\title{
Conflict-Free Evacuation Route Planner
}

\author{
Roxana Herschelman \\ rohriniuc2014@fau.edu \\ Florida Atlantic University \\ Boca Raton, Florida
}

\author{
KwangSoo Yang \\ yangk@fau.edu \\ Florida Atlantic University \\ Boca Raton, Florida
}

\begin{abstract}
Given a transportation network with node and edge capacity constraints, initial node occupancy, destination locations, and the conflict resolution parameter $k$, the Conflict-Free Evacuation Route Planner (CF-ERP) problem finds evacuation routes that can minimize the evacuation time and the number of movement conflicts along the routes. CF-ERP is important for many societal applications, such as evacuation management and preparation in case of natural or man-made disasters. The problem is computationally challenging due to the large size of the transportation network and the constraints. Related work has considered the evacuation routing problem either solely as a network flow optimization problem or a conflict minimization problem, but not both. In this paper, we propose novel approaches that can produce evacuation routes to minimize the evacuation time and the number of movement conflicts. Experiments and a case study on real-world datasets from Florida show the effectiveness and efficiency of the proposed approaches.
\end{abstract}

\section{CCS CONCEPTS}

-Information systems $\rightarrow$ Information systems applications; Geographic information systems; • Theory of computation $\rightarrow$ Network flows; Routing and network design problems;

\section{KEYWORDS}

Evacuation Route Planning, Constrained optimization, Spatial Graph Algorithms

\section{ACM Reference Format:}

Roxana Herschelman and KwangSoo Yang. 2019. Conflict-Free Evacuation Route Planner. In 27th ACM SIGSPATIAL International Conference on Advances in Geographic Information Systems (SIGSPATIAL '19), November 5-8, 2019, Chicago, IL, USA. ACM, New York, NY, USA, 4 pages. https: //doi.org/10.1145/3347146.3359102

\section{INTRODUCTION}

Evacuation route planning is a critical component for identifying the safest and most efficient routes in the wake of man-made or natural disasters (e.g., terrorist acts, hurricanes, and nuclear accidents). The main goal of evacuation route planning is to minimize the evacuation time for all expected residents in the evacuation zone. In 2018, there were 15 named storms, including eight hurricanes,

Permission to make digital or hard copies of part or all of this work for personal or classroom use is granted without fee provided that copies are not made or distributed for profit or commercial advantage and that copies bear this notice and the full citation on the first page. Copyrights for third-party components of this work must be honored.

For all other uses, contact the owner/author(s).

SIGSPATIAL '19, November 5-8, 2019, Chicago, IL, USA

(C) 2019 Copyright held by the owner/author(s)

ACM ISBN 978-1-4503-6909-1/19/11.

https://doi.org/10.1145/3347146.3359102 of which two were declared major (Category 3, 4, or 5) [5]. One major issue during these storms was a mass evacuation in which people blindly relied on the shortest routes without considering potential risks (e.g., traffic congestion, movement-conflicts, etc.), causing major delays. To minimize the congestion on roads when the traffic flow surpasses the capacity of the road network a simple shortest routing (e.g., Google Maps, Waze, etc.) is not adequate. In addition, traffic delay and car accidents at an intersection are some of the most important factors for traffic congestion during massive evacuation.

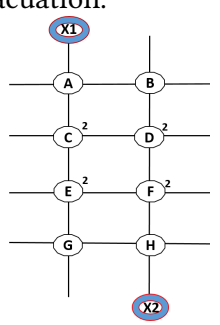

(a) CF-ERP Input

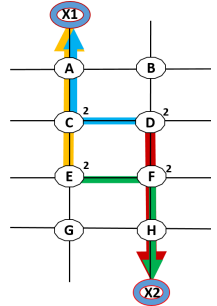

(b) Route Plan for minimizing the evacuation time

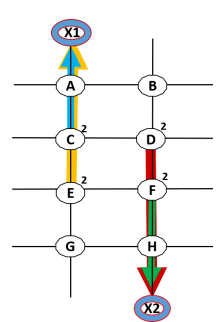

(c) CF-ERP Output
Figure 1: Example of Input and Output of CF-ERP

To remedy these issues, we propose a new problem, namely Conflict-Free Evacuation Route Planner (CF-ERP). The goal of CFERP is to minimize movement conflicts in evacuation routes and decrease the evacuation time by relieving congestion. Figure 1(a) shows an example input of CF-ERP, consisting of a graph with 8 graph-nodes $(A \ldots H)$ and two exits $(X 1$ and $X 2)$. Assume that the capacity of a road is one vehicle per time unit (i.e., 1$)$ and the travel time for each road segment is a unit of time (i.e., 1). Assume that nodes $C, D, E, F$ have 2 evacuees that need to be given instructions as to which is the best route to one of the exits. Figure 1(b) depicts one possible routing of evacuees to minimize the evacuation time. Each route is represented by a different color (i.e., Yellow, Blue, Red, and Green). As can be seen in this example, nodes $C$ and $F$ are a movement conflict point even though the evacuation time can be minimized. These conflict points may cause frequent stops, unnecessary delay, and vehicle accidents. An example of how routes can be changed to minimize the movement conflict is illustrated in Figure 1(c). In this example, evacuees starting at or passing through nodes $A, C$, and $E$ are routed only to exit $X 1$ (i.e., Yellow and Blue colored routes) while those from nodes $D, F$, and $H$ only follow routes that go to exit $X 2$ (i.e., Red and Green colored routes). This type of output is desirable because it lacks movement conflict and is easy to follow by the evacuees.

The CF-ERP problem is important for critical applications such as evacuation planning and management. CF-ERP ensures the safety of people by providing conflict-free routes to access exits in an overcrowded area. CF-ERP can be used for large event planning such as football games, large concerts, etc. 
Problem Definition: In our formulation of the CF-ERP problem, a transportation network is represented and analyzed as a directed graph composed of nodes $N$ and edges $E$. Each node represents a spatial location in geographic space (e.g., road intersections), which can be used as a proxy for locations of citizens or residences. Each edge between two nodes represents a road segment and has a travel distance and capacity (i.e., individuals/cars per time unit). A route schedule is a series of steps (nodes) that evacuees follow to reach the exits.

The $C F-\operatorname{ERP}(N, E, C, T, n, k)$ problem can be formally defined as follows:

Input: A transportation network $G$ with:

- a set of graph-nodes $N$, and a set of edges $E$,

- a set of positive integer capacity constraints $C: E \rightarrow Z^{+}$,

- a set of non-negative real travel times of edges $T: E \rightarrow R_{0}^{+}$,

- the total number of evacuees and their initial location, $n$ and

- the conflict resolution parameter $k$ (percentage of conflicts to be solved)

Output: An evacuation plan consisting of a set of origin-destination routes and a scheduling of evacuees on each route.

Objectives:

- Minimize the movement conflicts of the evacuees on routes.

- Minimize the total evacuation time.

\section{Constraints:}

- Evacuation plan observes the capacity constraints of the network.

- Limited amount of computer memory.

Definition 1. The conflict resolution parameter $k$ represents the percentage of nodes for which conflicts are solved for the whole transportation network throughout the whole evacuation time period.

Definition 2. A movement conflict is defined as the point of intersection between two routes that do not have the same exits.

Related Work: Current literature on ERP can be categorized into two main groups: (1) network flow optimization techniques that provide an optimal (or near-optimal) solution to minimize the evacuation time [1, 3, 6, 7], and (2) traffic engineering approaches that aim to minimize the movement conflicts [2]. However, these approaches are not suitable for optimizing two objectives simultaneously (i.e., minimization of the evacuation time and conflicts). By contrast, we propose a novel approach that can produce evacuation routes to minimize the evacuation time and the number of movement conflicts.

\section{PROPOSED APPROACHES FOR CF-ERP}

In this section, we introduce the Suffix-Tree Conflict Minimization (STCM) method to eliminate the movement conflicts among evacuation routes. Our proposed approaches use suffix trees to identify the movement conflicts and reduce these conflicts.

\subsection{Suffix Tree Conflict Minimization}

The main idea of STCM is to utilize historical information about the nodes in the transportation network. STCM gathers this information by using an existing network flow optimization method (i.e. Capacity Constrained Route Planner (CCRP) [4]). CCRP's output is a scheduling of evacuees on routes. An example of such route plan is shown in Figure 2. Figure 2(a) shows the visual representation of routes. Figure 2(b) shows a scheduling of evacuees on routes and their evacuation time (i.e., arrival time). Each evacuation route is represented as a series of steps (i.e., nodes) that include the arrival time at the node and the next destination node. In this example, the movement conflict takes place at nodes $C$ and $F$ due to the incoming traffic from nodes $D$ and $E$. The STCM method alleviates the traffic congestion by reducing this type of conflict.

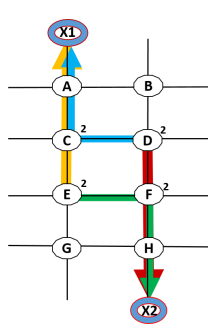

(a) Visual Representation of Routes

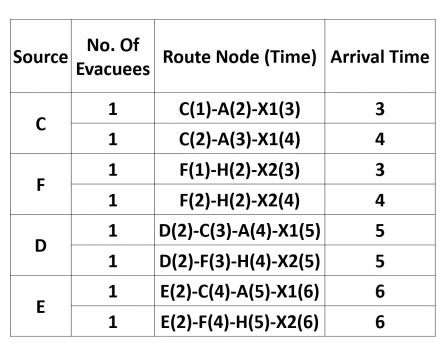

(b) Scheduling of Evacuees on Routes
Figure 2: Evacuation Plan Generated By Network Flow Optimization Techniques

The second main idea of STCM is to use suffix trees to reduce conflict. First, we construct a suffix tree for each of the destinations based on the routing schedule. Figure 3(a) illustrates the construction of the suffix trees from Figure 2(b). Second, we identify the nodes that are in more than one suffix tree (see Figure 3(b)). In this example, nodes $D$ and $E$ are located in both suffix trees, leading to the movement conflicts (see red-colored circles). Third, we modify these suffix trees by assigning nodes $D$ and $E$ to one of the suffix trees (see Figure 3(c)). Fourth, we construct the suffix trees that have no conflicting points (see Figure 3(d)). Finally, new evacuation routes are generated based on the modified suffix trees (see Figure 4).

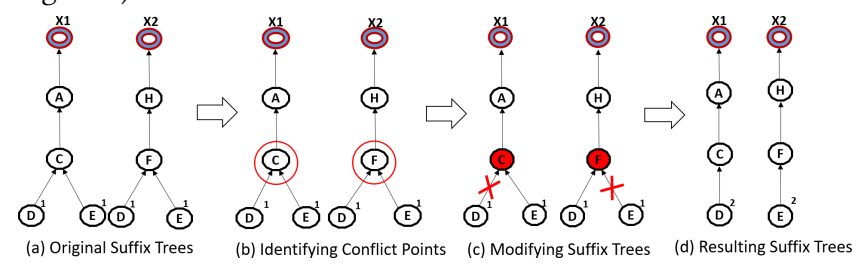

Figure 3: Suffix Tree Conflict Minimization

In this example, nodes $D$ and $E$ are no longer causing movement conflicts because their evacuation paths do not intersect anymore. Figure 4(a) shows that all evacuees from node $E$ only follow routes to exit $X 1$ (i.e., Blue and Yellow colored arrows) and evacuees from node $D$ only follow routes to exit $X 2$ (i.e., Red and Green colored arrows).

Given a transportation network in the form of a graph $G$, the total number of evacuees and their initial location, and the conflict resolution parameter $k$, STCM follows the following steps: Identify major congestion points by gathering historical data (i.e., routes and schedules of evacuees on these routes). The terminal node of the route is considered an exit from the endangered zone. For each exit, a suffix tree is created. Each node in a route is linked through an edge in the suffix tree to which it historically sent traffic to (i.e., evacuees used these nodes to reach the terminal node). We 


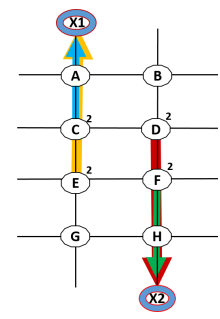

(a) Visual Representation of Routes

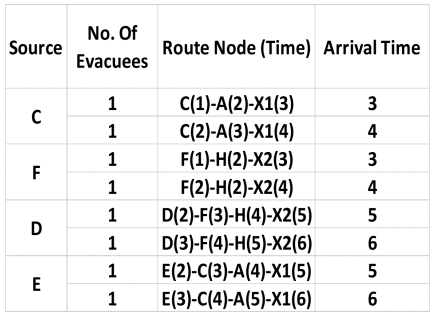

(b) Scheduling of Evacuees on Routes
Figure 4: Output of CF-ERP

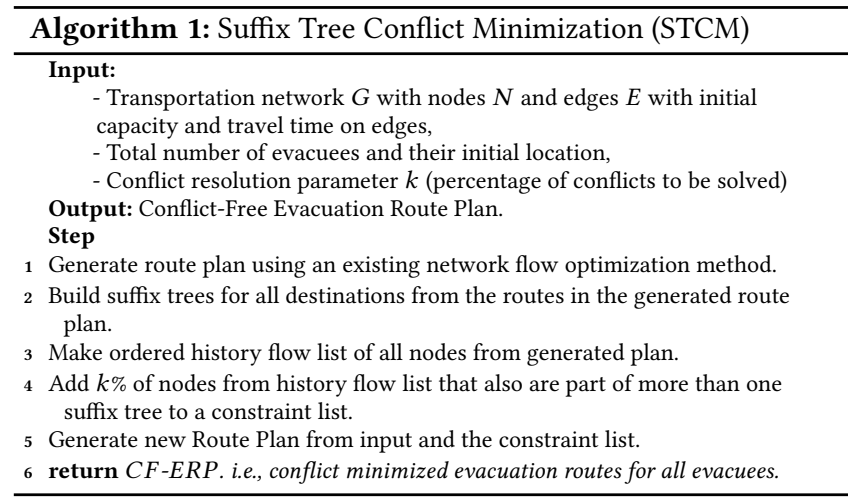

define the history of an evacuation node as the total number of evacuees that passed through that particular node throughout the duration of the evacuation. Once all routes have been added to the corresponding suffix trees, each node is classified based on its history. We then build a list of the history nodes that are also part of more than one suffix tree. This history list is then sorted in decreasing fashion. To alleviate congestion, STCM selects $k \%$ of nodes in this list. STCM restricts $k \%$ selected nodes to send evacuees to one of the suffix trees. In the process of generating a new route plan, the selected nodes will only send evacuees to one exit, unless no such route is possible. This constraint minimizes crisscross traffic and the waiting time, therefore reducing high congestion points.

The main steps of STCM are shown in Algorithm 1. STCM starts with generating a route plan (Line 1). Then, it builds suffix trees (Line 2). STCM orders all the nodes based on their history (Line 3) At line 4, STCM adds $k \%$ of the nodes that are also part of more than one suffix tree to the constraint list. STCM then generates a new route plan using the given input and the list of constraints.

The major issue in creating a CF-ERP is the new route plan generation. For this, we present two approaches: History based Suffix Tree Conflict Minimization (HSTCM), and Online Suffix Tree Conflict Minimization (OSTCM).

\subsection{History Based Suffix Tree Conflict Minimization}

The main idea of HSTCM is to identify the assignment of a node to the suffix tree that can minimize total evacuation time. HSTCM checks all possible assignments of the nodes in the constraint list to any of the suffix trees it has previously sent traffic flow to. Algorithm 2 presents the pseudo-code for the HSTCM algorithm. For
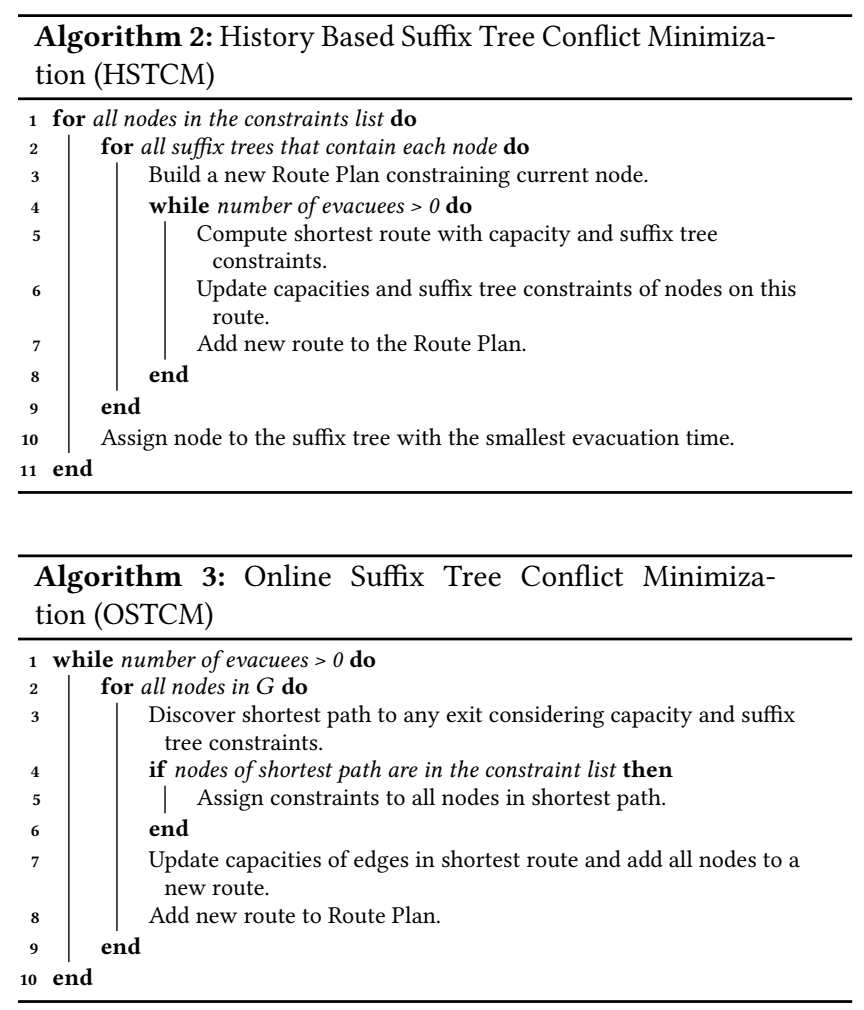

all the constraints of a node, HSTCM builds a route plan with the constraint such that the current node can send flow only to the current suffix tree (Line 3). For all the evacuees in the transportation network, HSTCM computes the shortest route considering available capacities and suffix tree constraints (Line 5). After that, it updates all the capacities and the suffix tree constraints of the edges in the shortest route (Line 6) and adds the new route to the Route Plan (Line 7). After all route plans have been computed for all suffix trees, HSTCM selects the route plan with the least evacuation time (Line 10) until all nodes in the $k \%$ history flow list have been processed.

\subsection{Online Suffix Tree Conflict Minimization}

Although HSTCM significantly reduces movement conflicts between evacuees, it may not be applicable for sizable road networks (e.g., the state of Florida map). Thus, we propose a more scalable algorithm, namely Online Suffix Tree Conflict Minimization (OSTCM), that makes use of the same data structure (i.e., the suffix trees). Algorithm 3 presents the pseudo-code for OSTCM. OSTCM computes shortest available routes considering the capacity constraints of the network as well as the suffix tree constraints at Line 3. OSTCM updates the suffix tree constraints if any of the nodes are in the $k \%$ list of constraints (Lines 4-6). The capacity constraints of the edges in the shortest path are updated at Line 7. A new route $R$ is added to the route plan (Line 8).

\section{EXPERIMENTAL EVALUATION}

In this section, we present the experiment design and an analysis of the experimental results. Mainly, we compared the overall performance of three algorithms: CCRP, HSTCM, and OSTCM. The 
comparison metrics used are: (1) total evacuation time, (2) the average number of movement conflicts within the route plans generated, and (3) total CPU run time. Figure 5 shows our experimental setup. All the algorithms were implemented using Java 1.8 with a $16 \mathrm{~GB}$ memory run-time environment on a machine running Windows 10 with $16 \mathrm{~GB}$ of RAM.

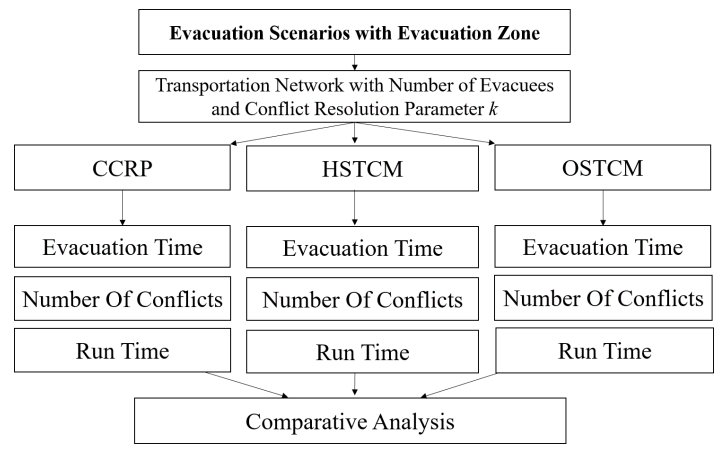

Figure 5: Experiment Layout

3.0.1 Effect of the Number of Evacuees. The first set of experiments evaluated the effect of the number of evacuees on algorithm performance. The experiments were evaluated on a Florida network with 2,718 nodes. We fixed the number of destinations to 26 and $k$ to 10 . The number of evacuees was increased from 2,500 to 30,000 . Figure 6(a) shows that as the number of evacuees increases, OSTCM outperforms the other two methods. Figure 6(b) shows that the increase in the number of evacuees does not impact the run time significantly. The effect of the population on the evacuation time of the three methods is shown in Figure 6(c). As the number of evacuees increases, the estimated evacuation time also increases.

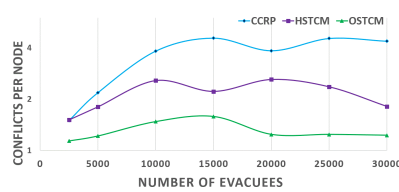

(a) Number of Conflicts

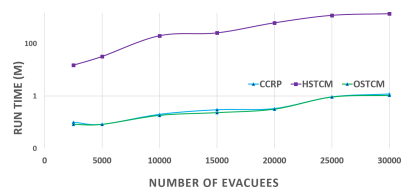

(b) Run Time

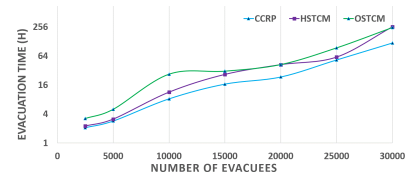

(c) Evacuation Time

Figure 6: Effect of the Number of Evacuees $(n=2,718, k=10$, and $d=26$ )

3.0.2 Effect of the Conflict Resolution Parameter $k$. A second experiment measures the influence of the conflict resolution parameter $k$ on the performance of the three analyzed algorithms. We used the same Florida transportation network with 26,920 evacuees, 2,718 nodes, and 26 destinations. $k$ was assigned to be $2 \%, 5 \%, 10 \%$, $20 \%$, and $100 \%$ respectively. Figure 7 (a) shows that as $k$ increases, OSTCM and HSTCM show a significant drop in the amount of conflicts they generate within their routes. Figure 7(b) shows that OSTCM has the smallest execution time overall. The execution time is not directly impacted by the increase of $k$. Figure 7 (c) shows the analysis of the effect of $k$ on the total evacuation time. HSTCM and OSTCM show that the evacuation time increases when $k$ is between 0 and 20 .

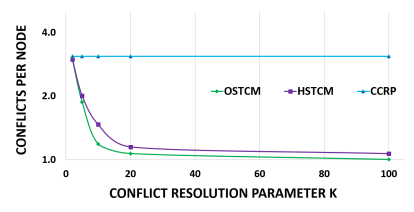

(a) Number of Conflicts

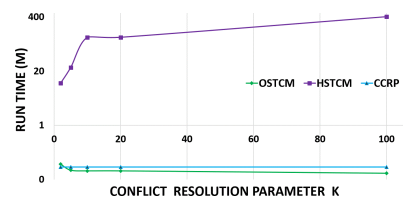

(b) Run Time

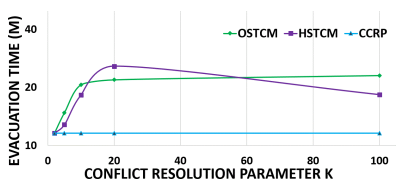

(c) Evacuation Time

Figure 7: Effect of the Conflict Resolution Parameter $(p=26,920, n=2,718$, and $d=26)$

\section{CONCLUSION AND FUTURE WORK}

We presented the problem of creating a Conflict-Free Evacuation Route Plan (CF-ERP). An important application of CF-ERP is generating route plans for emergency situations that minimize the movement conflict and the total evacuation time. Creating CF-ERP is challenging because of the large size of the transportation network, the capacity constraints of the network, the movement conflict between routes, and the limited amount of computer memory available. In this paper, we describe two approaches to solve the CF-ERP problem, namely History based Suffix Tree Conflict Minimization (HSTCM) and Online Suffix Tree Conflict Minimization (OSTCM). Both these approaches make use of the suffix tree data structure. Experiments using road data sets from Florida demonstrate that the proposed algorithms significantly decrease movement conflict during evacuation.

\section{ACKNOWLEDGMENTS}

We would like to thank the National Science Foundation under Grant No. 1844565.

\section{REFERENCES}

[1] RK Ahujia, Thomas L Magnanti, and James B Orlin. 1993. Network flows: Theory, algorithms and applications. New fersey: Rentice-Hall (1993).

[2] Thomas J. Cova and Justin P. Johnson. 2003. A network flow model for lane-based evacuation routing. Transportation Research Part A: Policy and Practice 37, 7 (2003), 579 - 604. https://doi.org/10.1016/S0965-8564(03)00007-7

[3] Bruce Hoppe and Tardos. 1994. Polynomial Time Algorithms for Some Evacuation Problems.. In SODA, Vol. 94. 433-441.

[4] Qingsong Lu, Betsy George, and Shashi Shekhar. 2005. Capacity Constrained Routing Algorithms for Evacuation Planning: A Summary of Results. In Advances in Spatial and Temporal Databases, Claudia Bauzer Medeiros, Max J. Egenhofer, and Elisa Bertino (Eds.). Springer Berlin Heidelberg, Berlin, Heidelberg, 291-307.

[5] National Oceanic and Athmospheric Administration (NOAA). 2018. Destructive 2018 Atlantic hurricane season draws to an end. (2018). Retrieved May 27, 2019 from https://www.noaa.gov/media-release/ destructive-2018-atlantic-hurricane-season-draws-to-end

[6] Shashi Shekhar, KwangSoo Yang, Venkata MV Gunturi, Lydia Manikonda, Dev Oliver, Xun Zhou, Betsy George, Sangho Kim, Jeffrey MR Wolff, and Qingsong Lu. 2012. Experiences with evacuation route planning algorithms. International fournal of Geographical Information Science 26, 12 (2012), 2253-2265.

[7] KwangSoo Yang, Venkata MV Gunturi, and Shashi Shekhar. 2012. A dartboard network cut based approach to evacuation route planning: A summary of results. In International Conference on Geographic Information Science. Springer, 325-339. 